\title{
Graph Routing Problem Using Euler's Theorem and Its Applications
}

\author{
Hashnayne Ahmed \\ Department of Mathematics, University of Barishal, Barishal, Bangladesh \\ Email address: \\ hashnayneahmed17@gmail.com \\ To cite this article: \\ Hashnayne Ahmed. Graph Routing Problem Using Euler's Theorem and Its Applications. Engineering Mathematics. \\ Vol. 3, No. 1, 2019, pp. 1-5. doi: 10.11648/j.engmath.20190301.11
}

Received: May 16, 2019; Accepted: June 17, 2019; Published: June 26, 2019

\begin{abstract}
In this modern era, time and cases related to time is very important to us. For shortening time, Eulerian Circuit can open a new dimension. In computer science, social science and natural science, graph theory is a stimulating space for the study of proof techniques. Graphs are also effective in modeling a variety of optimization cases like routing protocols, network management, stochastic approaches, street mapping etc. Konigsberg Bridge Problem has seven bridges linked with four islands detached by a river in such a way that one can't walk through each of the bridges exactly once and returning back to the starting point. Leonard Euler solved it in 1735 which is the foundation of modern graph theory. Euler's solution for Konigsberg Bridge Problem is considered as the first theorem of Graph Theory which gives the idea of Eulerian circuit. It can be used in several cases for shortening any path. From the Konigsberg Bridge Problem to ongoing DNA fragmentation problem, it has its applications. Aiming to build such a dimension using Euler's theorem and Konigsberg Bridge Problem, this paper presents about the history of remarkable Konigsberg Bridge Problem, Euler's Explanation on it, an alternative explanation and some applications to Eulerian Circuit using graph routing and Fortran Coding of it.
\end{abstract}

Keywords: Arc Routing, Konigsberg Bridge Problem, Graph Theory, Euler's Theorem Applications, Fortran Coding

\section{Introduction}

This interesting story starts in $17^{\text {th }}$ century in Konigsberg, Northern Germany. It has a vital role in the history of Graph Theory and Network Theory. Some citizens of Konigsberg town wanted to go for a walk in the seven bridges of town by passing each bridges for once. But they failed to find out a way for walking in the bridges for once and that's why the called Leonard Euler, famous mathematician at that time. [1,2]

Euler was in trouble to give a solution to the people of Konigsberg. But however it made the discovery of a branch of mathematics named Graph Theory. Euler described that if and only if every vertices with even number of degrees makes a circuit (same starting and ending point) then it is possible to pass each edges for exactly once and then it is named after him Eulerian Path or Eulerian Circuit. He also described two examples for solving Konigsberg Bridge Problem. In first example, he solved the problem for eight bridges. And in another, he solved it for sixteen bridges. [3, 4]

Further, Graph Routing has vast application in our day-to- day life. From the starting with postman letter delivery to VLSI (very large scale integration), it has root functioning. Focusing on Euler's Theorem these problem can easily be solved, which will be very effective for us to solve our time and anything related to it like cost, energy etc.

Konigsberg Bridge problem can also be solved using six bridges. The four partitions of Konigsberg town (by Pregel River) could also be joined with these six bridges for passing the bridges exactly once. Then it satisfies Euler's Theorem with finite number of routes possible.

\section{Graph Routing Problem}

We may define the graph routing problem by the definition of Eulerian Path (or Circuit). A graphical problem including an Eulerian circuit will be called a Graph Routing Problem or Arc Routing Problem. [5].

Definition 1: (Eulerian Circuit)

An Eulerian circuit in a graph is a sequence of edges that begins at a vertex of the graph and travels from vertex to vertex by crossing every edges for once and ends at the same 
vertex. $[6,7]$

For examples, we may consider the following graph:

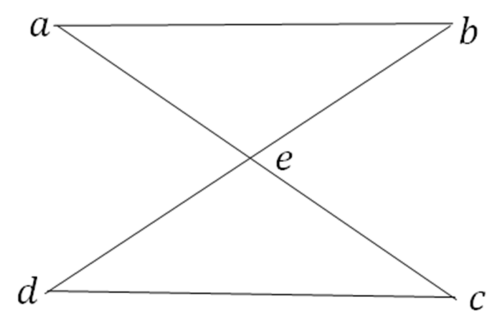

Figure 1. Eulerian Circuit (The graph has an Euler circuit named aecdeba).

For short, we will call Eulerian Circuit as $E C$ in this paper. The Bridges of Konigsberg is an example of a Graph Routing Problem. There are other similar type applications of graph routing: $[5,8]$

a. Optical Network connections use a specific way once.

b. Very Large Scale Integration (VLSI) on circuit board use the route once.

c. A Garbage collector must visit every houses garbage for once.

d. An Airplane traveler will travel from Dhaka to New York using a route for once with a cheap expenditure of money and time.

e. A postman deliver letters in a shortest possible way to every houses once known as Chinese Postman Problem.

\section{Leonard Euler's Generalization on Graph Theory}

Euler states that if there are no vertices with an odd number of degrees in a graph (undirected) then one can complete an Eulerian path or Eulerian Circuit in that graph means crossing every edges for once. [3]

\section{Simplifications on Euler's Theorem}

Actually, Euler Theorem is based on the simple trick that, if we consider a region where an object comes and also it is given that the object must left. Here the objects incoming way and outgoing way must be different.

Definition 2: (Degree of a vertex)

The degree of a vertex of a graph is the number of edges incident with it except that a loop at a vertex contributes twice to the degree of that vertex. The degree of a vertex $v$ is denoted by $\operatorname{deg}(v)$ or $\operatorname{deg} v .[6,9]$

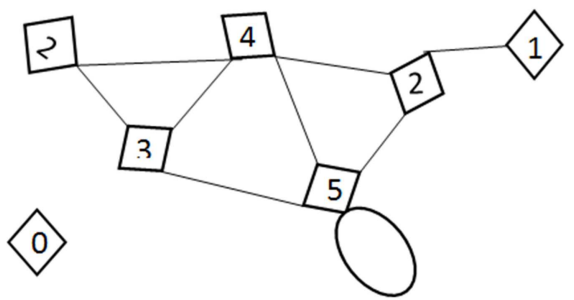

Figure 2. A graph with vertices labeled by degree.
So if we consider the regions as vertex and the path as edges then for crossing the edges only once the number of edges connected to each vertex must be two. In the same manner, if the objects repeat its travelling process for once again then it is $2+2=4$ edges to a vertex.

Proceeding in this way, we see that the number of edges connected to each vertex must be of even numbers like $2,4,6,8,10,12, \ldots$ for crossing the edges only once.

Again we see that for each vertex the number of incoming edges of the subject is equal to the number of outgoing edges. So for a vertex with even number of edges, the number of times appearing of edges is half of the degree $(\operatorname{deg} v)$ of that vertex.

\section{Konigsberg Bridge Problem Solution and Leonard Euler's Explanation}

This problem is a famous problem in the history of graph theory and there is a famous story behind this graph routing problem. Actually, an important division of mathematics, graph theory got its root from this problem.

Konigsberg (latterly named Kaliningrad) was the capital of German East Prussia. In 1254, King Ottoker II founded it. The following artwork is the figure of the town at that time.

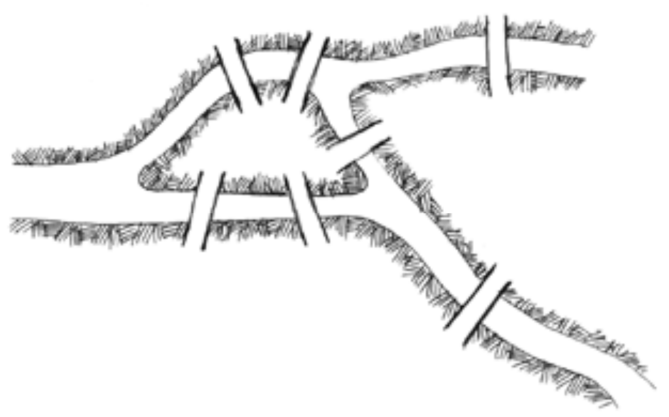

Figure 3. Konigsberg Bridge Problem.

The City, Konigsberg was divided into four parts in land by the river Prezel. The island Kneiphol was connected to other lands by bridges. A total number of 7 bridges were built to travel between these land areas.

As riverside area, soon it became popular to the citizens for walking. Some citizens wondered that, is it possible for them to walk along these bridges for exactly once at each bridge? But they could not get a solution to the problem. And soon it becomes a popular unsolvable problem to the people. To solve the problem they knocked Leonard Euler since Konigsberg was not too far from St. Petersburg (home of famous mathematician Leonard Euler).

On August 1735, Euler presented a paper named 'Solutio problematis ad geometriam situs pertinentis'. On that paper, Euler described solution to the Konigsberg bridge problem and pointed out graphically that if there are no regions with an odd number of landmasses then the called walking journey (routing graph) could be possible. After that the citizens of Konigsberg decided to build an extra bridge between land $B$ and $C .[1,2,10]$ 


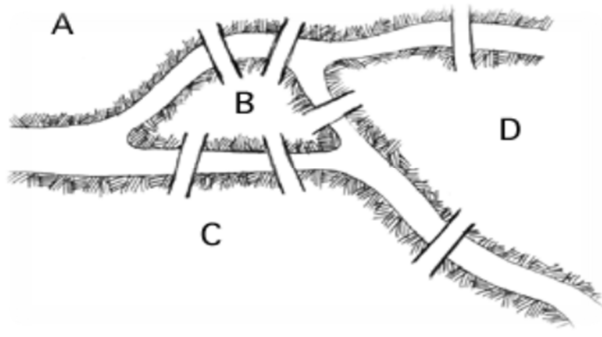

Figure 4. Konigsberg Bridge Problem (labeled).

\section{Alternative Solution to the Konigsberg Bridge Problem}

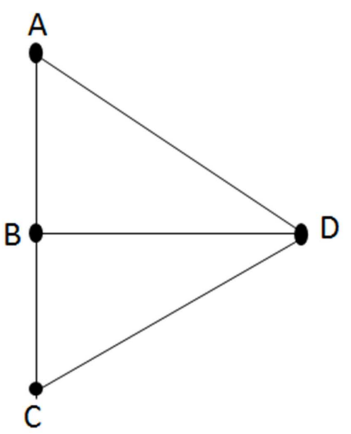

Figure 5. Konigsberg Bridge graphical model.

Here

Number of Bridges $=7$

Number of Bridges minus two and then plus one $=(7-$ 2) $+1=6$

So we may conclude,

Table 1. Region-wise Degree.

\begin{tabular}{lllll}
\hline Region & $\boldsymbol{A}$ & $\boldsymbol{B}$ & $\boldsymbol{C}$ & $\boldsymbol{D}$ \\
\hline Degree & 2 & 4 & 2 & 4 \\
\hline
\end{tabular}

Thus in the above figure, degrees for each region is even. And thus it satisfies the Euler's Theorem. So it can be a solution to the Konigsberg Bridge Problem.

We may describe the solution in details by the following figure:

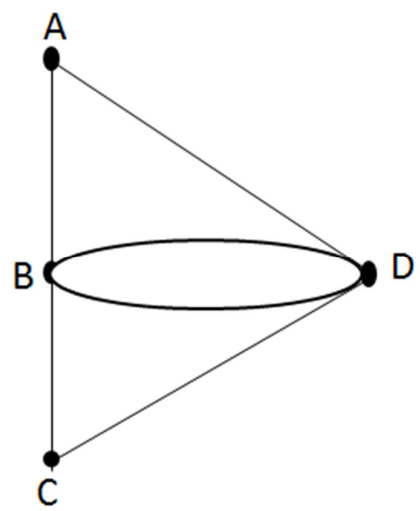

Figure 6. Solution of Konigsberg problem with six bridges.
If we choose the given vertex's one by one, For $A$,

Number of edges incoming, $I_{A}=1 ;\left(E_{D A}\right)$

Number of edges outgoing, $O_{A}=1 ;\left(E_{B A}\right)$

For $B$,

Number of edges incoming, $I_{B}=2 ;\left(E_{A B}, E_{D B}\right)$

Number of edges outgoing, $O_{B}=2 ;\left(E_{B C}, E_{B D}\right)$

For $C$,

Number of edges incoming, $I_{C}=1 ;\left(E_{B C}\right)$

Number of edges outgoing, $O_{C}=1 ;\left(E_{C D}\right)$

For $D$,

Number of edges incoming, $I_{D}=2 ;\left(E_{C D}, E_{B D}\right)$

Number of edges outgoing, $O_{D}=2 ;\left(E_{D B}, E_{D A}\right)$

Here at all vertices, number of edges incoming and outgoing are equal and the sum of incoming and outgoing are all even numbers. It ends there where it started. Thus, we get a Eulerian Circuit here and so the routing problem has been solved.

\section{Airplane Route Problem}

Suppose, a businessman have to visit in four cities of Bangladesh for his business purpose. He prefers air routes because of short time arrival. He also has an assignment for making a list of rating of services of the six air service providers in those regions. Air services in these regions have a form of following routes in the graph below:

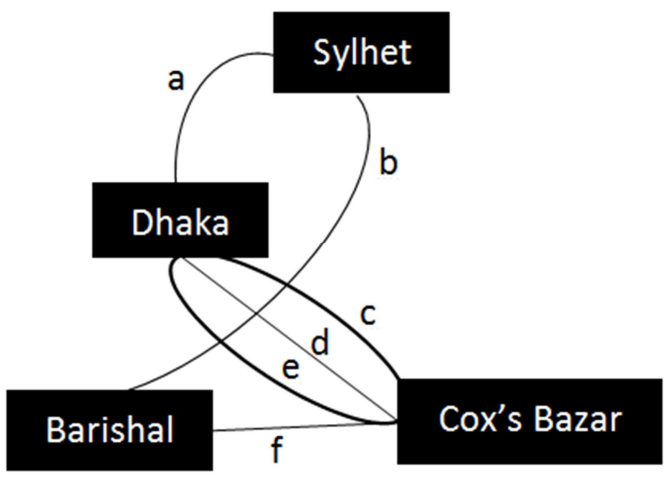

Figure 7. Graph Routing Problem.

Is it possible for the businessman to start from Dhaka and travelling the four cities with the six airways and then return back to Dhaka in purpose of business and assignment?

Solution

Here, there are four places to travel or four vertices. Now we will check for the vertices that the Euler theorem is satisfied or not.

For Dhaka,

Degree is 4 , which is even.

For Sylhet,

Degree is 2, which is even.

For Barisal,

Degree is 2, which is even.

For Cox's Bazar,

Degree is 4 , which is even.

That is, all the four vertices has even degree. So by Euler 
theorem is satisfied and hence it is possible for the businessman to start from Dhaka and travelling the four cities with the six airways and then return back to Dhaka in purpose of business and assignment.

\section{Fortran Coding}

program eulerian_circuit_checking

implicit none

integer::n

open $(11$, file='inputvalue.dat')

open $(12$, file='outputvalue.dat')

write $(*, *)$ 'Type the sum of degrees of all vertices in the file named inputvalue.dat..'

$\operatorname{read}(11, *) n$

if(n.eq.0)then

write $(12,20)$

20 format(1x,'To be an Eulerian Circuit total degree must be greater than zero.')

else if $(\bmod (n, 2)==0)$ then

write $(12,30)$

30 format(1x,'Its an Eulerian Circuit')

else

write $(12,40)$

40 format(1x,'Its not an Eulerian Circuit')

end if

end program

\section{Implication}

Finally we may get some implications regarding the discussions above.

Theorem 1. The minimum number of edges for an Eulerian circuit is the number of vertices itself.

Proof:

For Eulerian Circuit $(E C)$, each vertices should have an even number of degrees.

Define, $v$ for vertices and $e$ for edges. Then,

For $v=1$, the circuit is a loop with minimum edge is, $e=1(\operatorname{but} \operatorname{deg}(v)=2)$

For $v=2$, the Eulerian circuit forms with minimum edge is, $e=2$
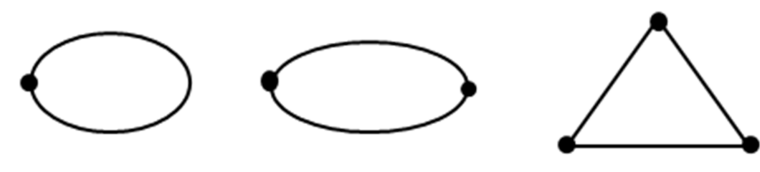

Figure 8. Eulerian Circuit with minimum edges.

In a similar manner, we can easily prove that the minimum number of edges for forming an Eulerian Circuit is the number of vertices itself.

Remarks. Since for Eulerian Circuit, degrees of each vertex is even so the total degree of the circuit must be even.

Theorem 2. If the number of edges for a circuit with minimum edges Eulerian Circuit $(\operatorname{minEC})$ is increased by 2
( 1 for loops) or multiple of 2, then it still forms an Eulerian Circuit.

Proof:

Let us suppose for an Eulerian Circuit with minimum edges, number of edges is $m$.

Then if we increase $m$ by of 2 , let $m^{*}=m+2 n$, where $n=1,2,3, \ldots \ldots \ldots$

We have to prove that $m^{*}$ edges also forms an Eulerian Circuit.

Since $m$ edges forms Eulerian Circuit, so for $=1, m^{*}=$ $m+2$; which is also an Eulerian Circuit as extra two edges adds an even number of degrees to any vertex.
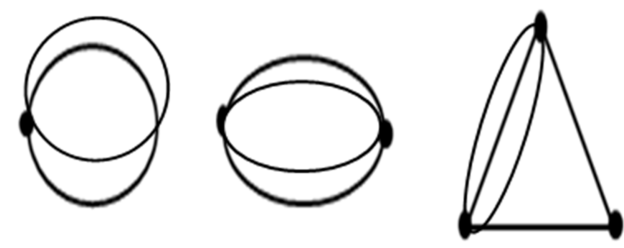

Figure 9. Eulerian Circuits.

In a similar manner we can see for $n=2,3, \ldots \ldots \ldots$, extra edges add even degrees to any vertex which satisfies the Euler's Theorem for.

Now for loop, each loop adds an even number of degrees to the vertex. So adding of one by one loop also ensures the condition of Eulerian Circuit.

For $m^{*}=2 m$, it also increases the degree in even number.

\section{Conclusion}

This paper shows the opportunities of shortening time and cases related to time like saving energy, low cost etc. The traditional graph routing problem has applications like: Optical network connection, Very large scale Integration on circuit board, Chinese Postman Problem [11], Kambi Kolam (a traditional interesting floor designs used in south India) [5] etc. It is also used in DNA recovery for reaching optimized category [5] The study of these applications starts with the history of Konigsberg Bridge Problem. Euler's Theorem is the foundation on many real world problem solving techniques. Which is the incentive behind Graph Theory. This paper introduces an alternative solution for Konigsberg Bridge Problem and opens door with more affordable and accurate ways for routing problem like airplane route, circuit board integration, stochastic optimization, travelling etc. using Eulerian Circuit.

\section{References}

[1] Gurjar, M. Applications of Euler's Theorem.

[2] Sanabria, S. Königsberg Bridge Problem.

[3] Euler, L. (1741). Solutio problematis ad geometriam situs pertinentis. Commentarii academiae scientiarum Petropolitanae, 128-140. 
[4] Shields, R. (2012). Cultural topology: The seven bridges of Königsburg, 1736. Theory, Culture \& Society, 29 (4-5), 43-57.

[5] Ismail, A. S., Hasni, R., \& Subramanian, K. G. (2009). Some applications of Eulerian graphs. International Journal of Mathematical Science Education, 2 (2), 1-10.

[6] Rosen, K. H., \& Krithivasan, K. (2012). Discrete mathematics and its applications: with combinatorics and graph theory. Tata McGraw-Hill Education.

[7] Gross, J. L., \& Yellen, J. (2005). Graph theory and its applications. Chapman and Hall/CRC.

[8] Dror, M. (Ed.). (2012). Arc routing: theory, solutions and applications. Springer Science \& Business Media.
[9] Biggs, N., Lloyd, E. K., \& Wilson, R. J. (1986). Graph Theory, 1736-1936. Oxford University Press.

[10] Paoletti, T. (2011). Leonard Euler's solution to the Königsberg bridge problem. URL http://www.

maa.org/press/periodicals/convergence/leonard-eulers-

solution-to-the-konigsberg-bridge-problem. (Cited on pages 9 and 10).

[11] Eiselt, H. A., Gendreau, M., \& Laporte, G. (1995). Arc routing problems, part I: The Chinese postman problem. Operations Research, 43 (2), 231-242. 